\title{
Effect of Ligusticum Chuanxiong on Hypoxic Pulmonary Hypertension in Rats and Its Mechanism
}

\author{
Congcong Zhang1, Xiaofei Chen ${ }^{2}$, Wantie Wang ${ }^{3 *}$ \\ ${ }^{1}$ Department of Pharmacy, Zhejiang Pharmaceutical College, Ningbo, China \\ ${ }^{2}$ Department of Respiratory and Critical Care Medicine, The Affiliated People's Hospital of Ningbo University, Ningbo, \\ China \\ ${ }^{3}$ Department of Pathophysiology, Wenzhou Medical University, Wenzhou, China \\ Email: *wwt@wmu.edu.cn
}

How to cite this paper: Zhang, C.C., Chen, X.F. and Wang, W.T. (2022) Effect of Ligusticum Chuanxiong on Hypoxic Pulmonary Hypertension in Rats and Its Mechanism. Journal of Biosciences and Medicines, 10, $20-26$

https://doi.org/10.4236/jbm.2022.102003

Received: October 8, 2021

Accepted: January 27, 2022

Published: January 30, 2022

Copyright $\odot 2022$ by author(s) and Scientific Research Publishing Inc. This work is licensed under the Creative Commons Attribution International License (CC BY 4.0).

http://creativecommons.org/licenses/by/4.0/

\begin{abstract}
Objective: To explore the mechanism of Chuanxiong in alleviating hypoxic pulmonary hypertension in rats by inhibiting pulmonary vascular remodeling. Methods: Thirty healthy and clean male SD rats weighing $(180-220) \mathrm{g}$ were randomly divided into three groups $(\mathrm{n}=10)$ : normoxia group $(\mathrm{n})$, hypoxia group $(\mathrm{H})$ and Chuanxiong group $(\mathrm{L})$. Group $\mathrm{N}$ was fed in normoxic environment, and the other two groups were fed in hypoxic $\left(9 \% 11 \% \mathrm{O}_{2}\right)$ environment for 4 weeks, 8 h/D, 6 days a week. Rats in group L were gavaged with Ligusticum chuanxiong solution diluted with normal saline at the concentration of $300 \mathrm{mg} / \mathrm{kg}$, and rats in group $\mathrm{H}$ were gavaged with equal volume of normal saline. After 4 weeks, the mean pulmonary artery pressure was measured. After pulmonary perfusion, the right ventricular free wall and left ventricle plus ventricular septum were taken to measure the right ventricular hypertrophy index. The changes of pulmonary morphology and ultrastructure were observed under light microscope. Results: Compared with group $\mathrm{n}$, the average pulmonary artery pressure and right ventricular hypertrophy index in the other two groups increased, and the thickening of pulmonary vascular wall was obvious under microscope $(\mathrm{P}<0.01)$; compared with group $\mathrm{H}$, the above changes in group $\mathrm{L}$ were reduced $(\mathrm{P}<0.05)$. Conclusion: Ligusticum chuanxiong can relieve pulmonary artery pressure in rats by inhibiting pulmonary vascular remodeling.
\end{abstract}

\section{Keywords}

Ligusticum Chuanxiong, Hypoxic Pulmonary Hypertension 


\section{Introduction}

Pulmonary arterial hypertension (PAH) is a group of diseases that can cause remodeling of pulmonary microvascular structure, increase pulmonary vascular resistance, and finally cause right heart failure, with high disability rate and mortality [1]. PAH is a common disease used to describe malignant diseases associated with elevated pulmonary artery pressure (greater than or equal to $25 \mathrm{mmHg}$ at rest or greater than or equal to $30 \mathrm{mmHg}$ during exercise) [2]. In recent years, scientists have made great efforts to explore $\mathrm{PAH}$, but they have not completely cured PAH. Current studies believe that pulmonary vascular remodeling is one of the main pathological changes of $\mathrm{PAH}$.

Pulmonary vascular remodeling includes myosis around non myogenic normal arteries, thickening of muscular artery wall caused by proliferation and hypertrophy of smooth muscle cells, deposition and increase of extracellular matrix proteins. Pulmonary vascular remodeling is a process of common thickening of pulmonary artery intima, media and adventitia. Such thickening is formed by the proliferation or hypertrophy of fibroblasts, smooth muscle cells, endothelial cells, collagen, elastin and other main cells in each layer [3]. Studies have shown that endothelial cells in the intimal layer of blood vessels can migrate from in situ and transform into mesenchymal cells or smooth muscle cells, which become endothelial mesenchymal transdifferentiation [4]. In animal models, the wall thickening caused by smooth muscle cell proliferation is reversible to some extent [5]. Under the action of a series of links, pulmonary vascular remodeling causes pulmonary arteriole wall thickening and lumen stenosis, resulting in decreased vascular compliance, increased right ventricular blood flow load and increased pulmonary artery pressure, which eventually leads to pulmonary hypertension and right heart failure, and even death in severe cases.

Ligusticum chuanxiong Hort is a kind of traditional Chinese medicine plant, which is often used to promote blood circulation and relieve body pain. It has a good therapeutic effect on some diseases such as coronary heart disease, cerebral thrombosis, cor pulmonale, heart failure and so on. It is found that Ligusticum chuanxiong has pharmacodynamic effects in protecting vascular endothelium, reducing red blood cell aggregation, antioxidant stress and antiplatelet [6]. In addition, Ligusticum chuanxiong has the effect of calcium antagonist, which can expand coronary vessels, reduce pulmonary artery pressure and achieve the purpose of treating cor pulmonale, but there are few studies on pulmonary vascular remodeling of chuanqiong at present. Therefore, this experiment aims to explore whether chuanqiong can alleviate the occurrence and progress of pulmonary hypertension by inhibiting pulmonary vascular remodeling, so as to provide new treatment ideas and methods for clinical prevention and treatment of pulmonary hypertension.

\section{Material}

\subsection{Animals}

Thirty healthy and clean male SD rats, weighing $180 \mathrm{~g}$ - $220 \mathrm{~g}$, were provided by 
the animal experiment center of Wenzhou Medical University. The animal license No: scxk (Zhejiang) 2015-0009.

\subsection{Reagents and Drugs}

Chuan Qiong (China); 4\% paraformaldehyde (Solarbio, Beijing); PE conduit (Australia); Ordinary microscope (Nikon, Japan); Orthostatic fluorescence microscope (Nikon, Japan); Microscope cover glass (Shitai, Jiangsu, China).

\subsection{Experimental Grouping and Model Preparation}

Thirty rats were randomly divided into three groups $(\mathrm{n}=10)$ : normoxia group $(\mathrm{n})$, hypoxia group $(\mathrm{H})$ and Chuanxiong group (L). Group $\mathrm{N}$ was fed in normoxic environment for 4 weeks, and the other two groups were fed in hypoxic (9\% $11 \% \mathrm{O}_{2}$ ) environment for 4 weeks, $8 \mathrm{~h} / \mathrm{D}, 6$ days a week. Rats in group $\mathrm{L}$ were gavaged with Ligusticum chuanxiong solution diluted with normal saline at the concentration of $300 \mathrm{mg} / \mathrm{kg}$ before warehousing, and rats in group $\mathrm{H}$ were gavaged with equal volume of normal saline before warehousing.

\subsection{Measure the Mean Pulmonary Arterial Pressure (mPAP) of Rats}

The rats were weighed and anesthetized by intraperitoneal injection of $0.3 \%$ Pentobarbital Sodium Solution $(1.0 \mathrm{ml} / 100 \mathrm{~g}$ body weight). After fixation, disinfect the rat's neck fur with alcohol, separate the subcutaneous tissue, expose and lift the external jugular vein, cut a "V" incision, and push the PE catheter connected to the pressure transducer inward along the incision until there is a small and regular pulmonary artery wave. The records are kept.

\subsection{Measurement of Right Ventricular Hypertrophy Index in Rats}

After mPAP was measured, the lung lobes and heart were perfused with normal saline, the heart was cut off and rinsed with PBS, the atrium and the root of large blood vessels were cut off along the atrioventricular sulcus, and the right ventricular free wall $(\mathrm{RV})$ and left ventricular + ventricular septum $(\mathrm{LV}+\mathrm{s})$ were retained. After the filter paper was dried, the $\mathrm{RV} /(\mathrm{LV}+\mathrm{s})$ was weighed with an electronic balance according to the formula, to determine the right ventricular hypertrophy index.

\subsection{HE Staining of Rat Lung Tissue}

The right middle lung lobe was soaked in $4 \%$ paraformaldehyde for 48 hours to 1 week. After dehydration, it was routinely paraffin sectioned and stained with he.

\subsection{Statistical Treatment}

The data are expressed by mean \pm standard deviation $( \pm s)$, statistical analysis is carried out by SPSS 19.0 software, and the normality test is carried out for the measurement data. One way ANOVA was used among multiple groups of sam- 
ples, LSD method was used for pairwise comparison of those with homogeneous variance, and Dunnett's T3 test was used for those with uneven variance.

\section{Results}

\subsection{Comparison of Mean Pulmonary Artery Pressure of Rats in Each Group}

After 4 weeks, compared with the rats in group $n$, the mPAP of the rats in the other two groups increased in varying degrees $(\mathrm{P}<0.01)$; compared with group $\mathrm{H}, \mathrm{mPAP}$ in group $\mathrm{L}$ decreased $(\mathrm{P}<0.01)$ (Figure 1 ).

\subsection{Comparison of Right Ventricular Hypertrophy Index in Each Group}

After 4 weeks, compared with group $\mathrm{n}$, the $\mathrm{RV} /(\mathrm{LV}+\mathrm{s})$ ratio of the other two groups increased in varying degrees $(\mathrm{P}<0.01)$; Compared with group $\mathrm{H}$, $\mathrm{RV} /(\mathrm{LV}+\mathrm{s})$ in group $\mathrm{L}$ showed a downward trend $(\mathrm{P}<0.01)$ (Figure 2$)$.

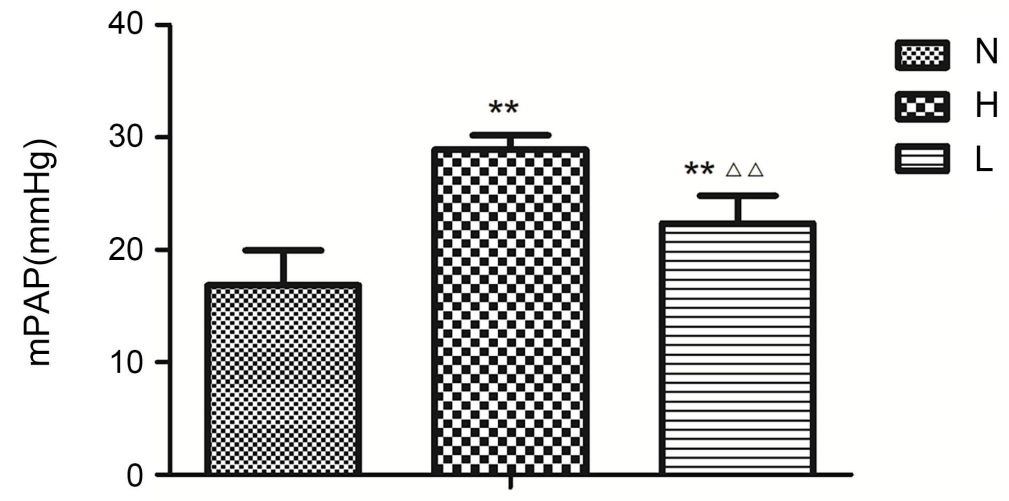

Group

Figure 1. Changes in mPAP of rats in each group. $(\bar{x} \pm s, \mathrm{n}=10) .{ }^{\star *} P<$ 0.01 vs. $\mathrm{N}$ group; ${ }^{\triangle} P<0.01$ vs. $\mathrm{H}$ group;

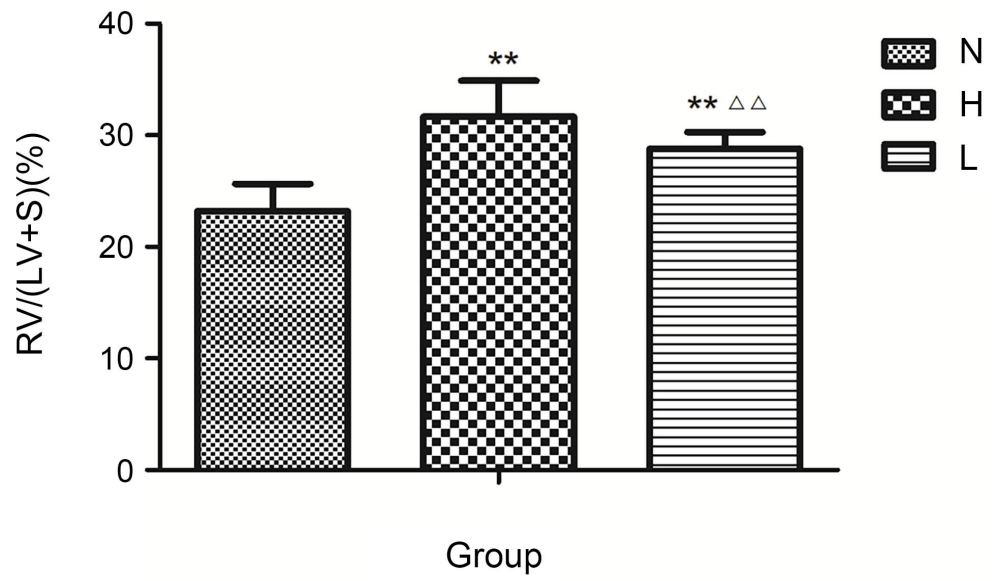

Figure 2. Changes in $\mathrm{RV} /(\mathrm{LV}+\mathrm{S})$ of rats in each group. $(\bar{x} \pm s, \mathrm{n}=10)$. ${ }^{* *} P$ $<0.01$ vs. $\mathrm{N}$ group; ${ }^{\triangle \triangle} P<0.01$ vs. HH group; 


\subsection{Light Microscopic Observation Results of Lung Tissue Morphology of Rats in Each Group}

Compared with group $\mathrm{n}$, pulmonary artery smooth muscle in the other two groups proliferated in varying degrees, WA/Ta increased $(\mathrm{P}<0.01), \mathrm{LA} / \mathrm{Ta}$ decreased $(\mathrm{P}<0.01)$; compared with group $\mathrm{H}$, the proliferation of pulmonary artery smooth muscle in group L decreased (Figure 3, Figure 4).

\section{Discussion}

$\mathrm{PAH}$ is a kind of progressive disease whose specific etiology and mechanism have not been clarified. Its main pathological changes include pulmonary vasoconstriction, pulmonary vascular remodeling and right ventricular hypertrophy [7]. PAH is difficult to cure, the disability rate is high, which greatly increases the social burden. Therefore, it is very important to do a good job in the prevention and treatment of PAH. PAH is often secondary to atrial septal defect [8], chronic obstructive pulmonary disease (COPD) [9], interstitial pulmonary fibrosis and related heart disease [10], and its pathogenesis is closely related to pulmonary vascular remodeling.

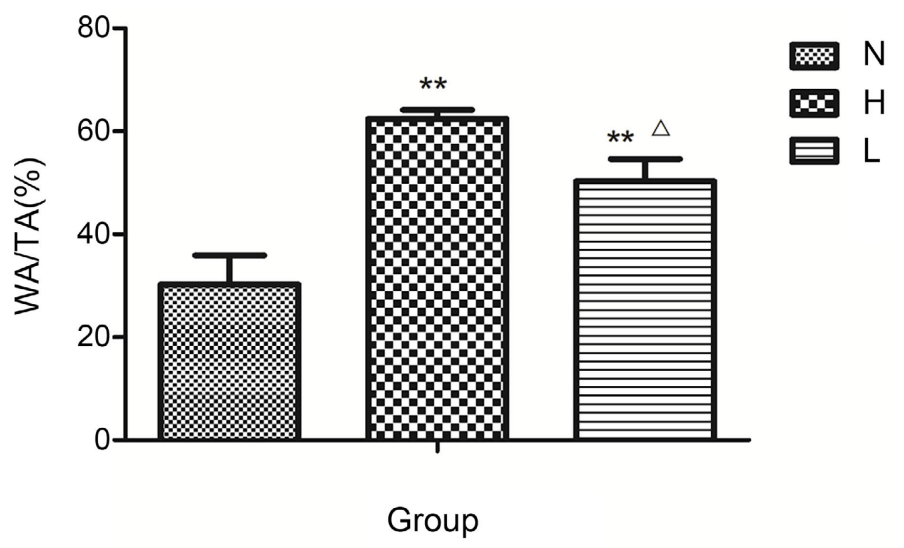

Figure 3. Comparison of WA/TA values of ratin each group. $(\bar{x} \pm s$, $\mathrm{n}=10)$.

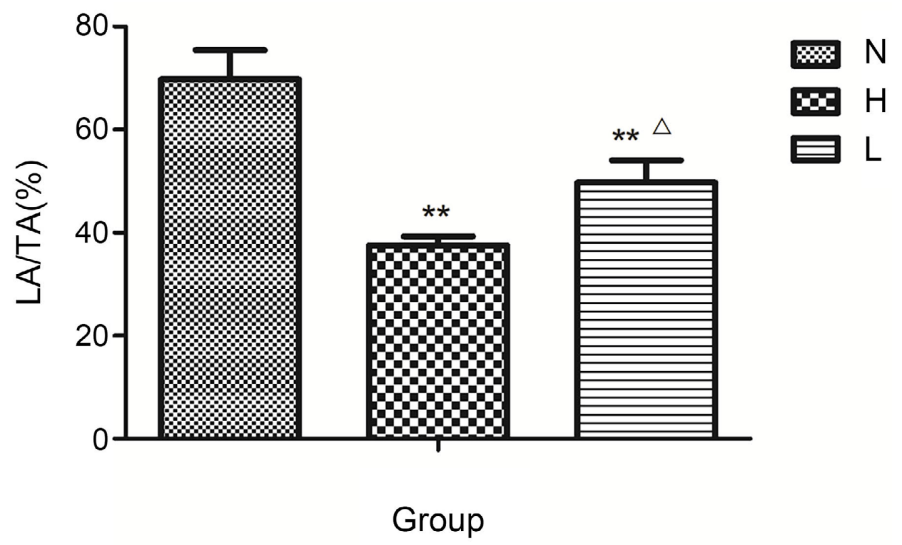

Figure 4. Comparison of LA/TA values of ratin each group. $(\bar{x} \pm s$, $\mathrm{n}=10) .{ }^{* *} P<0.01$ vs. $\mathrm{N}$ group; $\Delta P<0.05$ vs. $\mathrm{H}$ group. 
The effect of pulmonary vascular remodeling can affect the whole layer of vascular wall, which is characterized by inflammatory reaction in and around pulmonary vessels, hardening and thickening of pulmonary vascular wall, abnormal deposition of extracellular matrix, the expression of $\alpha$-Smooth muscle actin ( $\alpha$-SMA) increased, which led to the stenosis of vascular lumen and the increase of pulmonary artery pressure [11]. Pulmonary vascular remodeling involves many cytokines, such as VEGF, endothelin-1 (ET-1) and TGF- $\beta$, MMP plays a promoting role, while NO, prostacyclin and endostatin play a protective role. The molecular mechanism of pulmonary vascular remodeling is complex [12]. At present, there are three signal pathways studied more, namely mitogen activated protein kinase (MAPK) pathway, BMP/Smad signal pathway and Src/STAT3 pathway [13].

Although modern treatment has significantly improved the physiological function of PAH patients, its prognosis and mortality are still not optimistic. According to the basic mechanism and pathological changes of hypoxic pulmonary hypertension, chuanqiong was used for treatment. The equivalent dose of rats was converted according to the conversion rate of body surface area, and the concentration was $300 \mathrm{mg} / \mathrm{kg}$. The results showed that the activity state, diet and drinking water of the normal group were normal, while the model rats were obviously agitated and overreacted to slight stimulation, compared with group n, the pulmonary artery pressure, right ventricular hypertrophy index and pulmonary artery wall remodeling were significantly increased in the other two groups; compared with group $\mathrm{H}$, pulmonary artery pressure and right ventricular hypertrophy index decreased in group $\mathrm{L}$, which reversed pulmonary vascular remodeling to a certain extent.

In conclusion, Kawakami can reduce pulmonary artery pressure and reduce right ventricular hypertrophy, and its mechanism may be related to the inhibition of pulmonary vascular remodeling.

\section{Funding}

This study was supported by the Natural Science Foundation of Ningbo [2019A610350], A Project Supported by Scientific Research Fund of Zhejiang Provincial Education Department [Y202146228; Y202044087] and the Scientific research project of Zhejiang Pharmaceutical College [2019014].

\section{Conflicts of Interest}

The authors declare no conflicts of interest regarding the publication of this paper.

\section{References}

[1] Kooperkamp, H., Mehta, I., Fary, D., et al. (2017) Pulmonary Thromboendarterectomy for Pulmonary Hypertension before Considering Transplant. Ochsner Journal, 17, 66-70.

[2] Talwar, A., Sahni, S., Verma, S., et al. (2017) Exercise Tolerance Improves after 
Pulmonary Rehabilitation in Pulmonary Hypertension Patients. Journal of Exercise Rehabilitation, 13, 214-217. https://doi.org/10.12965/jer.1732872.436

[3] Bordenave, J., Tu, L., Savale, L., et al. (2019) New Insights in the Pathogenesis of Pulmonary Arterial Hypertension. Revue des Maladies Respiratoires, 36, 433-437. https://doi.org/10.1016/j.rmr.2019.03.003

[4] Zhang, H., Chang, H., Wang, L.M., et al. (2015) Effect of Polyelectrolyte Film Stiffness on Endothelial Cells during Endothelial-to-Mesenchymal Transition. Biomacromolecules, 16, 3584-3593. https://doi.org/10.1021/acs.biomac.5b01057

[5] Shu, D.Y., Butcher, E., Saint-Geniez, M., et al. (2020) EMT and EndMT: Emerging Roles in Age-Related Macular Degeneration. International Journal of Molecular Sciences, 21, 4271. https://doi.org/10.3390/ijms21124271

[6] Chen, Z., Zhang, C., Gao, F., et al. (2018) A Systematic Review on the Rhizome of Ligusticum Chuanxiong Hort. (Chuanxiong). Food and Chemical Toxicology, 119, 309-325. https://doi.org/10.1016/j.fct.2018.02.050

[7] Kim, D. and George, M.P. (2019) Pulmonary Hypertension. Medical Clinics of North America, 103, 413-423. https://doi.org/10.1016/j.mcna.2018.12.002

[8] Ali, M.A., Tazmin, T., Latif, T., et al. (2016) Incomplete Pentalogy of Cantrell-A Case Report. Mymensingh Medical Journal, 25, 153-157.

[9] Bajwa, A.A., Shujaat, A., Patel, M., et al. (2017) The Safety and Tolerability of Inhaled Treprostinil in Patients with Pulmonary Hypertension and Chronic Obstructive Pulmonary Disease. Pulmonary Circulation, 7, 82-88.

https://doi.org/10.1086/689291

[10] Lee, J.C., Kim, K.C., Choe, S.Y., et al. (2016) Reduced Immunoreactivities of B-Type Natriuretic Peptide in Pulmonary Arterial Hypertension Rats after Ranolazine Treatment. Anatomy \& Cell Biology, 49, 7-14. https://doi.org/10.5115/acb.2016.49.1.7

[11] Zhou, J.J., Li, H., Qian, Y.L., et al. (2020) Nestin Represents a Potential Marker of Pulmonary Vascular Remodeling in Pulmonary Arterial Hypertension Associated with Congenital Heart Disease. Journal of Molecular and Cellular Cardiology, 149, 41-53. https://doi.org/10.1016/j.yjmcc.2020.09.005

[12] Zhang, Y., Tang, J., Li, N., et al. (2021) Prenatal Hypoxia Induced ET B R Activation and Abnormal ROS Signalling in Pulmonary Artery Cells of Rat Offspring. Reproductive Toxicology, 105, 91-100. https://doi.org/10.1016/j.reprotox.2021.08.009

[13] Tian, H., Liu, L., Wu, Y., et al. (2021) Resistin-Like Molecule $\beta$ Acts as a Mitogenic Factor in Hypoxic Pulmonary Hypertension via the $\mathrm{Ca}^{2+}$-Dependent PI3K/Akt/mTOR and PKC/MAPK Signaling Pathways. Respiratory Research, 22, Article No. 8. https://doi.org/10.1186/s12931-020-01598-4 